Pacific Journal of Mathematics

RIESZ SETS AND A THEOREM OF BOCHNER 


\title{
RIESZ SETS AND A THEOREM OF BOCHNER
}

\section{H. Alan MacLean}

\begin{abstract}
Conditions are developed from which one may infer that a measurable subset $S$ of an LCA group $\hat{G}$ is a Riesz set, or in general, a small $p$ set. Subsets of inverse images of small $p$ sets under continuous homomorphisms which are small $q$ sets for some $q$ are investigated. These considerations yield extensions to LCA groups of Bochner's version of the F. and M. Riesz theorem.
\end{abstract}

The aim of this article is to develop criteria which, under appropriate circumstances, imply that a subset of an LCA group is a Riesz set, or in general, a small $p$ set. The conditions developed lead one rather naturally to consider the behavior of inverse images of Riesz sets under continuous homomorphisms, specifically to consider if or when the inverse image of a Riesz set, or a subset thereof, is again a Riesz set. Special emphasis is given to this aspect of the problem, and, in those cases where a solution is obtained, to certain applications which arise as a consequence.

The notion of a Riesz set has its origins in the F. and M. Riesz theorem, which provides the prototype for all Riesz sets, namely, the nonnegative integers $Z^{+}$. This renowned theorem states that if the Fourier transform $\hat{\mu}$ of a measure on $[0,2 \pi)$ vanishes off $Z^{+}$, then $\mu$ is absolutely continuous with respect to Lebesgue measure ([9], [10, 8.2.1]). By analogy, if $G$ is an LCA group and $S$ is a measurable subset of $\hat{G}$, then one calls $S$ a Riesz set if each measure $\mu \in M(G)$ whose transform $\hat{\mu}$ vanishes off $S$ is absolutely continuous with respect to Haar measure on $G$. Any measurable subset of $\hat{G}$ having finite Haar measure in $\hat{G}$ is a Riesz set, this being a consequence of the Inversion theorem ([5, 31.33]). Bochner's well-known generalization of the F. and M. Riesz theorem to $Z^{n}$ provides a multitude of nontrivial Riesz sets in $Z^{n}$ ([1, Theorem 5], [10, 8.2.5]). Loosely speaking, for $n=2$ this theorem states that the set of points in any "angle" in $Z \times Z$ of less than $\pi$ radians is a Riesz set. In particular, the Cartesian product $Z^{+} \times Z^{+}$of the Riesz set $Z^{+}$is again a Riesz set. As we shall see later, versions of Bochner's theorem continue to hold in the general group setting ("Bochner's theorem" in the sequel will refer to the theorem mentioned above, as opposed to the one having to do with positive-definite functions). 
In [3] Glicksberg shows for metrizable $G$ that if $h: \hat{G} \rightarrow R$ (the real line) is a nonconstant continuous homomorphism and if $S \subseteq h^{-1}\left(R^{+}\right)$is a closed subset of $\hat{G}$ such that $h^{-1}\left(x-R^{+}\right) \cap S$ has finite Haar measure in $\hat{G}$ for all $x \in R$, then $S$ is a Riesz set in $\hat{G}$. The proof is based on disintegration of measures and, as is pointed out, this technique yields variations of Bochner's theorem. It is also shown in [3] that if $S$ is a closed subset of $\hat{G}$ such that $S \cap(\gamma-S)$ has finite Haar measure for all $\gamma \in \hat{G}$, then $\mu * \mu$ is absolutely continuous whenever $\hat{\mu}$ vanishes off $S$. This last result provides motivation for the definition of a small $p$ set (see [2]), which will be given shortly. Again the proof utilizes disintegration of measures (for simplified proofs of the latter result which do not use disintegration see [4] and [7]). Recently, Yamaguchi [12] has established a version of Bochner's theorem valid for LCA groups. Specifically, he has shown that if $S_{1}$ is a Riesz set in $\hat{G}_{1}$ and $S_{2}$ is a small $p$ set in $\hat{G}_{2}$, then $S_{1} \times S_{2}$ is a small $p$ set in $\hat{G}_{1} \times \hat{G}_{2}$. The motivation for what follows stems from the articles [3] and [12]; in particular, an essential ingredient derives from the refinements of the disintegration technique of [3] found in [12]. We begin by setting notation and making appropriate definitions. We then state and prove our main theorems (Theorems 2 and 3), and derive a variety of consequences and applications.

Let $G$ be a locally compact abelian (LCA) group. We denote Haar measure on $G$ by $m_{G}$ (or simply $d x$, if no confusion is possible) and the dual group of $G$ by $\hat{G}$. If $H$ is a closed subgroup of $G$ we denote the annihilator of $H$ by $H^{\perp}=\{\gamma \in \hat{G}: \gamma(x)=1, x \in H\}$. We identify the duals of $G / H$ and $H$, as usual, with $H^{\perp}$ and $\hat{G} / H^{\perp}$, respectively. $C_{0}(G)$ is the space of continuous complex functions on $G$ which vanish at infinity, and $C_{00}(G)$ the subspace of $C_{0}(G)$ consisting of functions with compact suppport. $M(G)$ is the Banach algebra of bounded complex regular Borel measures on $G$. When considering individual measures $\mu \in M(G)$ we shall, when necessary, regard $\mu$ as being defined on the $\sigma$-algebra of all $\mu$-measurable subsets of $G$. We identify the ideal $M_{a}(G)$ of absolutely continuous measures in $M(G)$ with $L^{1}(G)$ via their Radon-Nikodym derivatives.

The Fourier (-Stieltjes) transform of $\mu \in M(G)$ is defined on $\hat{G}$ by

$$
\hat{\mu}(\gamma)=\int_{G} \overline{\gamma(x)} d \mu(x) .
$$

If $f \in L^{1}(|\mu|)$, then we often write $\mu(f)=\int_{G} f d \mu$ so that, for example, $\nu(\bar{\gamma})=\hat{\mu}(\gamma) . M_{0}(G)$ consists of those $\mu \in M(G)$ for which $\hat{\mu}$ vanishes at infinity. 
Let $G$ and $G_{1}$ be LCA groups and let $\rho: G \rightarrow G_{1}$ be a continuous homomorphism of $G$ into $G_{1}$. We denote the kernel of $\rho$ by $\operatorname{ker} \rho$ and let $\varphi$ denote the natural quotient map $\varphi: \hat{G} \rightarrow \hat{G} /(\operatorname{ker} \rho)^{\perp}$. The symbol $\varphi$ will always stand for this particular mapping unless specifically indicated otherwise. We denote the adjoint of $\rho$ by $\bar{\rho}$. Thus, $\bar{\rho}: \hat{G}_{1} \rightarrow \hat{G}$ is the continuous homomorphism defined by $\bar{\rho}(\chi)=\chi \circ \rho$. For $\mu \in M(G)$ we denote the image of $\mu$ with respect to $\rho$ by $\rho(\mu)$. The measure $\rho(\mu)$ belongs to $M\left(G_{1}\right)$ and satisfies

$$
\int_{G_{1}} f(y) d \rho(\mu)(y)=\int_{G} f \circ \rho(x) d \mu(x)
$$

for all bounded Borel functions $f: G_{1} \rightarrow C$ so that, in particular, $\rho(\mu) \hat{(\chi)}$ $=\hat{\mu}(\chi \circ \rho)=\hat{\mu}(\bar{\rho}(\chi))$ for all $\chi \in \hat{G}_{1}$.

Suppose now that $G_{1}$ is metrizable, and let $\mu \in M^{+}(G)$. Then we may "disintegrate" $\mu$ with respect to $\rho$ ([6, Chapter IX, in particular p. 154]). That is to say, there exists a map $\lambda: G_{1} \rightarrow M^{+}(G) ; y \rightarrow \lambda_{y}$, a so-called "disintegration" of $\mu$, with the following properties. where;

(a) $\lambda_{y}$ is concentrated on $\rho^{-1}(y)$ and $\left\|\lambda_{y}\right\| \leq 1 \rho(\mu)$-almost every-

(b) the map $y \rightarrow \lambda_{y}(g)$ is $\rho(\mu)$-measurable for each $g \in C_{00}(G)$, and

$$
\int_{G} g(x) f \circ \rho(x) d \mu(x)=\int_{G_{1}} f(y) \lambda_{y}(g) d \rho(\mu)(y)
$$

for all $g \in C_{00}(G)$ and $f \in C_{00}\left(G_{1}\right)$.

The following observations will be useful later. First, (b) and (c) continue to hold for all bounded Borel functions $g: G \rightarrow C$ and $f: G_{1} \rightarrow C$ (cf. [6, Proposition 6, p. 147]). Further, if (a) holds except for $y \in A$, where $\rho(\mu)(A)=0$, then we may redefine $\lambda_{y}$ to be 0 for each $y \in A$, so that (a) now holds everywhere, without affecting the validity of (b) and (c). Note, in particular, that we then have $\lambda_{y}=0$ for all $y \in G_{1} \backslash \rho(G)$, since $\lambda_{y}=0$ already for $y \in G_{1} \backslash A \cup \rho(G)$ by (a). In short, we may (and will) assume that (a) holds everywhere on $G_{1}$ with $\lambda_{y}=0$ for all $y \in$ $G_{1} \backslash \rho(G)$, and that (b) and (c) hold for all bounded Borel $g: G \rightarrow C$ and $f$ : $G_{1} \rightarrow C$.

Definition 1. Let $G$ be an LCA group and $p$ a positive integer. For $\mu \in M(G)$ set $\mu^{p}=\mu * \mu * \cdots * \mu$ ( $p$ times). A measurable subset $S \subseteq \hat{G}$ is called a small $p$ set in $\hat{G}$ provided $\mu^{p} \in L^{1}(G)$ whenever $\mu \in M(G)$ and $\hat{\mu} \equiv 0$ on the complement of $S$. A Riesz set is by definition a small 1 set. 
Here "measurable" means measurable with respect to Haar measure on $\hat{G}$. The following lemma is undoubtedly well-known, although we have no reference and so include a proof.

LEMMA 1. Let $G_{1}$ and $G_{2}$ be LCA groups and let $\beta$ : $G_{1} \rightarrow G_{2}$ be a 1-1 continuous homomorphism of $G_{1}$ into $G_{2}$. If $B \subseteq G_{1}$ and $m_{G_{1}}(B)=0$, then $m_{G_{2}}(\beta(B))=0$.

Proof. Let $H=\cup_{1}^{\infty} K_{n}, K_{n}$ compact, be an open $\sigma$-compact subgroup of $G_{1}$ which contains $B$. If $m_{G_{2}}(\beta(H))=0$, then $m_{G_{2}}(\beta(B))=0$ also. Thus, we may suppose $m_{G_{2}}(\beta(H)) \neq 0$. Then $m_{G_{2}}\left(\beta\left(K_{n}\right)\right)>0$ for some $n$ so $\beta\left(K_{n}\right)-\beta\left(K_{n}\right)$ contains a neighborhood of 0 in $G_{2}([5,20.17])$, whence $\beta(H)$ is open in $G_{2}$. Since $H$ is $\sigma$-compact, the restriction of $\beta$ to $H$ is then an open mapping onto $\beta(H)$, hence a topological isomorphism. Now $H$ is open, so Haar measure $m_{H}$ on $H$ is the restriction of $m_{G_{1}}$ to $H$. Consequently, $m_{H}(B)=0$, and since $\beta: H \rightarrow \beta(H)$ is a topological isomorphism, it follows that $m_{\beta(H)}(\beta(B))=0$. But $\beta(H)$ is open in $G_{2}$ so that once again $m_{\beta(H)}$ is simply the restriction of $m_{G_{2}}$ to $\beta(H)$. Therefore, $m_{G_{2}}(\beta(B))=0$.

Theorem 1. Let $G$ be a metrizable LCA group and let $S$ and $E$ be measurable subsets of $\hat{G}$ with $S \subseteq E$. Suppose $G_{1}$ is an LCA group and $\rho$ : $G \rightarrow G_{1}$ is a continuous homomorphism such that $\overline{\rho(G)}$ is metrizable. If

(i) $\bar{\rho}^{-1}(\gamma+S)$ is a small $q$ set in $\hat{G}_{1}$ for a dense set $D$ of $\gamma \in \hat{G}$, and

(ii) $\varphi(E)$ is a small $p$ set in $\hat{G} /(\operatorname{ker} \rho)^{\perp}$, then $S$ is a small pq set in $\hat{G}$.

We may, of course, take $S=E$ above. However, it is convenient to have the theorem stated in terms of $S$ and $E$ for use later in connection with inverse images of small $p$ sets, where we will take $E=h^{-1}(T)$.

Proof. Let $\mu$ be a measure in $M(G)$ such that $\hat{\mu}$ vanishes off $S$ and set $\nu=\rho\left(\left|\mu^{q}\right|\right)$. We begin by showing that

$$
\nu \in L^{1}\left(G_{1}\right) \text {. }
$$

Let $\gamma \in D$ and suppose $\chi \notin \bar{\rho}^{-1}(\gamma+S)$. Then $\chi \circ \rho-\gamma \notin S$ so

$$
\rho(\gamma \mu) \hat{)}(\chi)=\hat{\mu}(\chi \circ \rho-\gamma)=0 .
$$

By (i) the set $\bar{\rho}^{-1}(\gamma+S)$ is small $q$ in $\hat{G}_{1}$ and thus $\rho\left(\gamma \mu^{q}\right)=\rho(\gamma \mu)^{q} \in$ $L^{1}\left(G_{1}\right)$. It follows that if $Q=\sum_{1}^{n} c_{j} \gamma_{j}$ is a trigonometric polynomial with $\gamma_{j} \in D(1 \leq j \leq n)$, then $\rho\left(Q \mu^{q}\right) \in L^{1}\left(G_{1}\right)$. Since $D$ is dense in $\hat{G}$, the set

$$
T_{D}=\left\{\sum_{1}^{n} c_{j} \gamma_{j}: \gamma_{j} \in D, j=1, \ldots, n\right\}
$$


is dense in $L^{1}\left(\left|\mu^{q}\right|\right)$ (see [5, 31.4]), and we therefore choose a sequence $\left(Q_{n}\right)$ in $T_{D}$ such that $Q_{n} \mu^{q} \rightarrow\left|\mu^{q}\right|$. Then since $\left\|\rho\left(Q_{n} \mu^{q}\right)-\rho\left(\left|\mu^{q}\right|\right)\right\| \leq$ $\left\|Q_{n} \mu^{q}-\left|\mu^{q}\right|\right\|$, it follows that $\nu=\rho\left(\left|\mu^{q}\right|\right) \in L^{1}\left(G_{1}\right)$.

Now, let $H=\operatorname{ker} \rho$ and choose any open $\sigma$-compact subgroup $H_{0}$ of $G$ which contains supp $|\mu|$. Let $\rho_{0}$ be the restriction of $\rho$ to $H_{0}$ and set $\nu^{\prime}=\rho_{0}\left(\left|\mu^{q}\right|\right)$. Note that $\nu^{\prime}$ is concentrated on the $\sigma$-compact set $\rho\left(H_{0}\right)$.

The function $\rho_{0}: H_{0} \rightarrow \overline{\rho\left(H_{0}\right)}$ is a continuous homomorphism and $\overline{\rho\left(H_{0}\right)} \subseteq \overline{\rho(G)}$ is metrizable by hypothesis. We may therefore "disintegrate" $\left|\mu^{q}\right|$ with respect to $\rho_{0}$. Doing so we obtain measures $\left\{\lambda_{y}^{\prime}: y \in\right.$ $\left.\overline{\rho\left(H_{0}\right)}\right\} \subseteq M^{+}\left(H_{0}\right)$ such that (a)-(c) hold with $\rho, \mu, G$, and $G_{1}$ replaced by $\rho_{0},\left|\mu^{q}\right|, H_{0}$, and $\overline{\rho\left(H_{0}\right)}$. As noted in the remarks following (a)-(c), we may assume (b) and (c) hold for all bounded Borel $g: H_{0} \rightarrow C$ and $f$ : $\overline{\rho\left(H_{0}\right)} \rightarrow C$, and $\lambda_{y}^{\prime}$ is concentrated on $\rho_{0}^{-1}(y)=\underline{\rho^{-1}(y)} \cap H_{0}$ with $\left\|\lambda_{y}^{\prime}\right\| \leq 1$ for all $y \in \overline{\rho\left(H_{0}\right)}$. Furthermore, $\lambda_{y}^{\prime}=0$ if $y \in \overline{\rho\left(H_{0}\right)} \backslash \rho\left(H_{0}\right)$.

The extension $\omega$ of $\nu^{\prime}$ to $G_{1}(\omega(B))=\nu^{\prime}\left(B \cap \overline{\rho\left(H_{0}\right)}\right)$ is simply the measure $\nu=\rho\left(\left|\mu^{q}\right|\right)$. In particular, $\nu$ is concentrated on $\rho\left(H_{0}\right)$. For $y \in G_{1}$ define $\lambda_{y}^{\prime \prime} \in M^{+}(G)$ by

$$
\lambda_{y}^{\prime \prime}= \begin{cases}\lambda_{y}^{\prime} \text { extended to } G & \text { if } y \in \rho\left(H_{0}\right), \\ 0 & \text { if } y \in G_{1} \backslash \rho\left(H_{0}\right) .\end{cases}
$$

Next let $\mu^{q}=f_{0}\left|\mu^{q}\right|$, where $f_{0}$ is Borel with $\left|f_{0}\right|=1$, and set $\lambda_{y}=f_{0} \lambda_{y}^{\prime \prime} \in$ $M(G)$. Then, in view of the preceding paragraph, the following three properties hold for the family $\left\{\lambda_{y}: y \in G_{1}\right\} \subseteq M(G)$ :

$$
\begin{aligned}
& \lambda_{y} \text { is concentrated on } \rho^{-1}(y) \cap H_{0} \text { and }\left\|\lambda_{y}\right\| \leq 1 \text { for all } \\
& y \in G_{1}, \lambda_{y}=0 \text { if } y \in G_{1} \backslash \rho\left(H_{0}\right),
\end{aligned}
$$

$y \rightarrow \lambda_{y}(g)$ is $\nu$-measurable for each bounded Borel function $g: G \rightarrow C$, and

$$
\int_{G} g(x) f \circ \rho(x) d \mu^{q}(x)=\int_{G_{1}} f(y) \lambda_{y}(g) d \nu(y)
$$

for all bounded Borel $g: G \rightarrow C$ and $f: G_{1} \rightarrow C$.

For each $y \in \rho\left(H_{0}\right)$ let $y_{0}$ be a fixed element in the set $\rho^{-1}(y) \cap H_{0}$, and for each $y \in G_{1} \backslash \rho\left(H_{0}\right)$ set $y_{0}=0$. Define measures $\sigma_{y} \in M(G)$ by setting

$$
\sigma_{y}=\lambda_{y} * \delta_{-y_{0}} \quad\left(y \in G_{1}\right),
$$

where $\delta_{-y_{0}}$ is the point mass at $-y_{0}$. Then by (2)

$$
\text { supp } \sigma_{y} \subseteq H \cap H_{0}
$$

for all $y \in G_{1}$. 
Now, in view of (6) we may regard each $\sigma_{y}$ as being a measure in $M(H)$. Doing so we then claim that

$$
\left\{\begin{array}{l}
\text { there exists a subset } K \subseteq G_{1} \text { with } \nu\left(K^{c}\right)=0 \text { such that } \\
\hat{\boldsymbol{\sigma}}_{y}=0 \text { off } \varphi(E) \subseteq \hat{G} / H^{\perp} \text { for all } y \in K
\end{array}\right.
$$

To establish (7) we begin by observing that one may choose closed sets $K_{n} \subseteq \operatorname{supp} \nu(n \geq 1)$ such that for each $n$,

$$
\nu\left(K_{n}^{c}\right)<1 / n,
$$

(9) $\nu\left(K_{n} \cap V\right)>0$ for each open $V \subseteq G_{1}$ such that $K_{n} \cap V \neq \varnothing$,

and

$$
\begin{aligned}
& \text { the map } y \rightarrow \lambda_{y}(\hat{f}) \text { is continuous on } K_{n} \text { for each } \\
& f \in L^{1}(\hat{G}) \text {. }
\end{aligned}
$$

This may be seen as follows. $H_{0}$ is $\sigma$-compact and metrizable (since by hypothesis $G$ is) so $C_{0}\left(H_{0}\right)$ is separable. Then the subspace $A$ of $C_{0}(G)$ consisting of all $f \in C_{0}(G)$ with supp $f \subseteq H_{0}$ is also separable with, say, a countable dense set $\mathscr{F}$. For each $n \geq 1$ apply Luzin's theorem and (3) to find a closed set $K_{n} \subseteq \operatorname{supp} \nu$ such that (8) and (9) hold, and such that the mapping $y \rightarrow \lambda_{y}(f)$ is continuous on $K_{n}$ for each $f$ in the countable set $\mathscr{F}$. Then since $\mathscr{F}$ is dense in $A$ and the norms of the $\lambda_{y}$ are uniformly bounded, it follows that $y \rightarrow \lambda_{y}(f)$ is continuous on $K_{n}$ for all $f \in A$. Now let $f \in L^{1}(\hat{G})$. Since $H_{0}^{\perp}$ is compact, $\left(f * m_{H_{0}^{\perp}}\right) \hat{E} A$. Noting that $\lambda_{y}=\sigma_{y} * \delta_{y_{0}}$, and recalling that supp $\sigma_{y} \subseteq H_{0}$ and $y_{0} \in H_{0}$, we find that

$$
\begin{aligned}
\lambda_{y}\left(\left(f * m_{H_{0}^{\perp}}\right)^{\wedge}\right) & =\int_{G} \hat{f}\left(x+y_{0}\right) \xi_{H_{0}}\left(x+y_{0}\right) d \sigma_{y}(x) \\
& =\int_{G} \hat{f}\left(x+y_{0}\right) d \sigma_{y}(x)=\lambda_{y}(\hat{f}) .
\end{aligned}
$$

Consequently, $y \rightarrow \lambda_{y}(\hat{f})$ is continuous on $K_{n}$, from which it follows that (8) - (10) hold.

To complete the verification of (7) let $f \in L^{1}(\hat{G})$ with $\operatorname{supp} f \subseteq$ $\varphi^{-1}(\varphi(E))^{c}$. Let $\gamma \in \hat{G}$ and $\chi \in \hat{G}_{1}$. If $f(\gamma) \neq 0$, then since $S+\bar{\rho}\left(\hat{G}_{1}\right)$ $\subseteq E+H^{\perp}$, we must have $\gamma \notin S+\bar{\rho}\left(\hat{G}_{1}\right)$. Thus $\gamma-\chi \circ \rho \notin S$, so $\left.\left(\mu^{q}\right) \hat{(\gamma}-\chi \circ \rho\right)=0$. Therefore, using (4)

$$
\begin{aligned}
0 & =\int_{\hat{G}} f(\gamma)\left(\mu^{q}\right)^{\hat{G}}(\gamma-\chi \circ \rho) d \gamma=\int_{G} \int_{\hat{G}} f(\gamma) \overline{\gamma(x)} \chi \circ \rho(x) d \gamma d \mu^{q}(x) \\
& =\int_{G} \hat{f}(x) \chi \circ \rho(x) d \mu^{q}(x)=\int_{G_{1}} \chi(y) \lambda_{y}(\hat{f}) d \nu(y) .
\end{aligned}
$$


Since trigonometric polynomials are dense in $L^{1}(\nu)$ it follows from the calculation above that

$$
\int_{G_{1}} F(y) \lambda_{y}(\hat{f}) d \nu(y)=0
$$

for all $F \in L^{1}(\nu)$. But $y \rightarrow \lambda_{y}(\hat{f})$ belongs to $L^{\infty}(\nu)$ so we conclude that

$$
\lambda_{y}(\hat{f})=0 \quad \text { for } \nu \text {-almost all } y \in G_{1} .
$$

We now infer from (9) and (10) that, in fact,

$$
\lambda_{y}(\hat{f})=0 \quad \text { for all } y \in K_{n} \quad(n \geq 1) .
$$

Thus for $y \in K_{n}$,

$$
0=\lambda_{y}(\hat{f})=\int_{G_{1}} \hat{f}(x) d \lambda_{y}(x)=\int_{\hat{G}_{1}} f(\gamma) \hat{\lambda}_{y}(\gamma) d \gamma
$$

Since (11) holds for all $f \in L^{1}(\hat{G})$ with supp $f \subseteq \varphi^{-1}(\varphi(E))^{c}$ we have

$$
\hat{\lambda}_{y}(\gamma)=0 \quad \text { for all } \gamma \in \varphi^{-1}(\varphi(E))^{c} \text { and } y \in K_{n} \quad(n \geq 1)
$$

(note that it is implicit in (ii) that $\varphi(E)$, hence $\varphi^{-1}(\varphi(E)$ ) also, is measurable).

Now let $K=\cup_{1}^{\infty} K_{n}$. Then $\nu\left(K^{c}\right)=0$ by (8). Since supp $\sigma_{y} \subseteq H$, $\hat{\sigma}_{y}$ is constant on cosets of $H^{\perp}$. Moreover, if $\gamma+H^{\perp} \notin \varphi(E)$, then $\gamma \notin$ $\varphi^{-1}(\varphi(E))$, so if $y \in K,(5)$ and (12) show that

$$
\hat{\sigma}_{y}\left(\gamma+H^{\perp}\right)=\hat{\sigma}_{y}(\gamma)=\hat{\lambda}_{y}(\gamma) \gamma\left(y_{0}\right)=0 \text {. }
$$

Thus $\hat{\sigma}_{y}$ vanishes off $\varphi(E)$ for all $y \in K$ and this establishes the claim in (7).

By (ii) $\varphi(E)$ is small $p$ in $\hat{G} / H^{\perp}$, and by (7) $\hat{\sigma}_{y}=0$ off $\varphi(E)$ for all $y \in K$. Thus,

$$
\sigma_{y_{1}} * \cdots * \sigma_{y_{p}} \in L^{1}(H)
$$

whenever $y_{1}, \ldots, y_{p} \in K$ (see [11, Lemma 4]).

Let $G_{1}^{p}=G_{1} \times \cdots \times G_{1}(p$ times $)$ and define $\omega \in M(G)$ by

$$
\omega(f)=\int_{G p} \lambda_{y_{1}} * \cdots * \lambda_{y_{p}}(f) d(\nu \times \cdots \times \nu)\left(y_{1}, \ldots, y_{p}\right)
$$


for $f \in C_{0}(G)$ (it follows from (3) that the function $\left(y_{1}, \ldots, y_{p}\right) \rightarrow$ $\lambda_{y_{1}} * \cdots * \lambda_{y_{p}}(f)$ is $(\nu \times \cdots \times \nu)$-measurable for bounded Borel $f: G \rightarrow$ $C$; cf. [12]). This formula extends to all bounded Borel functions $f$ : $G \rightarrow C$. For $\gamma \in \hat{G}$ we have by (4) that

$$
\begin{aligned}
\hat{\omega}(\gamma) & =\int_{G P} \lambda_{y_{1}} * \cdots * \lambda_{y_{p}}(\bar{\gamma}) d(\nu \times \cdots \times \nu)\left(y_{1}, \ldots, y_{p}\right) \\
& =\prod_{1}^{p} \int_{G_{1}} \hat{\lambda}_{y_{j}}(\gamma) d \nu\left(y_{j}\right)=\prod_{1}^{p} \int_{G} \bar{\gamma} d \mu^{q}=\left(\mu^{p q}\right)^{\hat{n}}(\gamma) .
\end{aligned}
$$

Thus, $\omega=\mu^{p q}$.

Now, let $B$ be a Borel set in $G$ such that $m_{G}(B)=0$. Then there exists a Borel set $F \subseteq G / H$ such that

$$
m_{G / H}\left(F^{c}\right)=0,
$$

and

$$
m_{H}((B-x) \cap H)=\int_{H} \xi_{B}(x+h) d h=0 \quad \text { for all } x+H \in F .
$$

Let $\beta: G / H \rightarrow G_{1} ; \beta(x+H)=\rho(x)$ be the 1-1 continuous map induced on the quotient by $\rho$. Then by (15) and Lemma $1, m_{G_{1}}\left(\rho(G) \cap \beta(F)^{c}\right)=$ $m_{G_{1}}\left(\beta\left(F^{c}\right)\right)=0$. Since we know by (1) that $\nu^{p} \ll m_{G_{1}}$ and $\nu^{p}$ is concentrated on $\rho\left(H_{0}\right)$, it follows that $\nu^{p}\left(\beta(F)^{c}\right)=0$. Letting $\alpha$ : $G_{1}^{p} \rightarrow G_{1}$ be the map $\alpha\left(y_{1}, \ldots, y_{p}\right)=y_{1}+\cdots+y_{p}$, we thus find that

$$
(\nu \times \cdots \times \nu)\left(\alpha^{-1}(\beta(F))^{c}\right)=\nu^{p}\left(\beta(F)^{c}\right)=0 .
$$

Furthermore, since by (7) $\nu\left(K^{c}\right)=0$, we also have $(\nu \times \cdots \times \nu)\left(\left(K^{p}\right)^{c}\right)$ $=0$, and therefore that

$$
(\nu \times \cdots \times \nu)\left(\left(K^{p}\right)^{c} \cup \alpha^{-1}(\beta(F))^{c}\right)=0 .
$$

Next let $\left(y_{1}, \ldots, y_{p}\right) \in K^{p} \cap \alpha^{-1}(\beta(F))$. Let $y_{j 0}$ be the fixed element associated with $\sigma_{y_{j}}$ as in (5). Then we assert that

$$
\sigma_{y_{1}} * \cdots * \sigma_{y_{p}}\left(\left(B-\sum_{1}^{p} y_{j 0}\right) \cap H\right)=0 .
$$

For if all of the $y_{j}$ lie in $\rho\left(H_{0}\right)$, then, by definition, $y_{j 0} \in \rho^{-1}\left(y_{j}\right)(1 \leq j \leq p)$, and we must have $y_{10}+\cdots+y_{p 0}+H \in F$. Otherwise,

$$
\sum_{1}^{p} y_{j}=\sum_{1}^{p} \rho\left(y_{j 0}\right)=\beta\left(\sum_{1}^{p} y_{j 0}+H\right) \in \beta\left(F^{c}\right) \subseteq \beta(F)^{c},
$$


which contradicts that $\Sigma_{1}^{p} y_{j}=\alpha\left(y_{1}, \ldots, y_{p}\right) \in \beta(F)$, since $\left(y_{1}, \ldots, y_{p}\right) \in$ $\alpha^{-1}(\beta(F))$. Thus, $y_{1}^{0}+\cdots+y_{p 0}+H \in F$, so, by (16),

$$
m_{H}\left(\left(B-\sum_{1}^{p} y_{j 0}\right) \cap H\right)=0 .
$$

But each $y_{j} \in K$, so by (13) $\sigma_{y_{1}} * \cdots * \sigma_{y_{p}} \in L^{1}(H)$, and this together with the last line shows that (18) holds. If, on the other hand, some $y_{j} \notin \rho\left(H_{0}\right)$, then (2) and (5) show that $\sigma_{y_{j}}=0$ and (18) clearly holds. Thus, (18) holds for all $\left(y_{1}, \ldots, y_{p}\right) \in K^{p} \cap \alpha^{-1}(\beta(F))$.

Therefore, combining (14), (17), and (18) and recalling that the support of each $\sigma_{y}$ lies in $H$ we find that

$$
\begin{aligned}
\mu^{p q}(B)= & \int_{G P} \lambda_{y_{1}} * \cdots * \lambda_{y_{p}}\left(\xi_{B}\right) d(\nu \times \cdots \times \nu)\left(y_{1}, \ldots, y_{p}\right) \\
= & \int_{G P} \int_{G} \xi_{B}\left(x+\sum_{1}^{p} y_{j 0}\right) d\left(\sigma_{y_{1}} * \cdots * \sigma_{y_{p}}\right)(x) \\
& \times d(\nu \times \cdots \times \nu)\left(y_{1}, \ldots, y_{p}\right) \\
= & \int_{K^{p} \cap \alpha^{-1}(\beta(F))} \sigma_{y_{1}} * \cdots * \sigma_{y_{p}}\left(\left(B-\sum_{1}^{p} y_{j 0}\right) \cap H\right) \\
\times d(\nu \times \cdots \times \nu)\left(y_{1}, \ldots, y_{p}\right) & \\
& +\int_{\left[K^{p} \cap \alpha^{-1}(\beta(F))\right]^{c}} \sigma_{y_{1}} * \cdots * \sigma_{y_{p}}\left(\left(\begin{array}{r}
\left.\left.p-\sum_{1}^{p} y_{j 0}\right) \cap H\right) \\
\times d(\nu \times \cdots \times \nu)\left(y_{1}, \ldots, y_{p}\right)
\end{array}\right.\right. \\
= & 0+0=0 .
\end{aligned}
$$

Thus, $\mu^{p q} \in L^{1}(G)$ and the proof is complete.

The assumption that $G$ be metrizable in Theorem 1 may be eliminated, and also in some cases the requirement that $\overline{\rho(G)}$ be metrizable. This is carried out with the aid of the following Lemma, which reduces the general case in part to Theorem 1. First, some notation.

Let $G$ and $G_{1}$ be LCA groups and let $\rho: G \rightarrow G_{1}$ be a continuous homomorphism into $G_{1}$. If $\Gamma$ is a closed subgroup of $\hat{G}$, then we set $\Gamma_{1}=\bar{\rho}^{-1}(\Gamma)$ and denote the restriction of $\bar{\rho}$ to $\Gamma_{1}$ by $\bar{\rho}_{0}: \Gamma_{1} \rightarrow \Gamma . \bar{\rho}_{0}$ is the adjoint of the induced homomorphism $\rho_{0}: G / \Gamma^{\perp} \rightarrow G_{1} / \Gamma_{1}^{\perp}$ defined by $\rho_{0}\left(x+\Gamma^{\perp}\right)=\rho(x)+\Gamma_{1}^{\perp}$. We let $\varphi_{0}: \Gamma \rightarrow \Gamma /\left(\operatorname{ker} \rho_{0}\right)^{\perp}$ denote the natural quotient map. 
LEMMA 2. Notation as in the last paragraph. Let $S \subseteq E$ be measurable subsets of $\hat{G}$ which satisfy (i) and (ii) of Theorem 1 (we do not assume that either $G$ or $\overline{\rho(G)}$ is metrizable). Let $\Gamma$ be an open $\sigma$-compact subgroup of $\hat{G}$. Then

(i) $\bar{\rho}_{0}^{-1}(\gamma+S \cap \Gamma)$ is a small $q$ set in $\Gamma_{1}$ for a dense set $D^{\prime}$ of $\gamma \in \Gamma$, and

(ii) $\varphi_{0}(E \cap \Gamma)$ is a small $p$ set in $\Gamma /\left(\operatorname{ker} \rho_{0}\right)^{\perp}$. Consequently, if $\overline{\rho_{0}\left(G / \Gamma^{\perp}\right)}$ is metrizable, then $S \cap \Gamma$ is a small pq set in $\hat{G}$.

Proof. If $\gamma \in D \cap \Gamma=D^{\prime}$, then

$$
\bar{\rho}_{0}^{-1}(\gamma+S \cap \Gamma)=\bar{\rho}^{-1}(\gamma+S) \cap \Gamma_{1} .
$$

Since $\gamma \in D$, Theorem 1(i) shows that $\bar{\rho}^{-1}(\gamma+S)$, hence $\bar{\rho}^{-1}(\gamma+S) \cap \Gamma_{1}$ is also small $q$ in $\hat{G}_{1}$. Now, since $\Gamma_{1}$ is open in $\hat{G}_{1}$, a subset $T \subseteq \Gamma_{1}$ is small $q$ in $\hat{G}_{1}$ if and only if $T$ is small $q$ in $\Gamma_{1}$ ([11, Lemme 6]). Thus, $\bar{\rho}_{0}^{-1}(\gamma+S \cap \Gamma)$ is small $q$ in $\Gamma_{1}$ for each $\gamma \in D^{\prime}$, which is dense in $\Gamma$, so (i)' holds.

In order to establish (ii) ${ }^{\prime}$ we first note that since $\bar{\rho}$ is the adjoint of $\rho$, $\Gamma_{1}=\bar{\rho}^{-1}\left(\Gamma \cap \bar{\rho}\left(\hat{G}_{1}\right)\right)=\rho\left(\Gamma^{\perp}\right)^{\perp}$, and thus $\Gamma_{1}^{\perp}=\rho\left(\Gamma^{\perp}\right)\left(\rho\left(\Gamma^{\perp}\right)\right.$ is closed in $G_{1}$ since $\Gamma^{\perp}$ is compact). Hence, letting $\pi: G \rightarrow G / \Gamma^{\perp}$ be the quotient map, we have

$$
\operatorname{ker} \rho_{0}=\pi\left(\rho^{-1}\left(\Gamma_{1}^{\perp}\right)\right)=\pi\left(\Gamma^{\perp}+\operatorname{ker} \rho\right)=\pi(\operatorname{ker} \rho) .
$$

It follows easily from these last relations that $\left(\operatorname{ker} \rho_{0}\right)^{\perp}=\Gamma \cap$ $(\operatorname{ker} \rho)^{\perp}$. Thus our objective is to show that $\varphi_{0}(E \cap \Gamma)$ is a small $p$ set in $\Gamma / \Gamma \cap(\operatorname{ker} \rho)^{\perp}$.

Let $H=(\operatorname{ker} \rho)^{\perp}$. Now since $\Gamma$ is open and $\sigma$-compact in $\hat{G}$ we have $\Gamma / \Gamma \cap H \approx \Gamma+H / H([5,5.33])$. Specifically, the map $\alpha: \Gamma+H / H \rightarrow$ $\Gamma / \Gamma \cap H$ defined by $\alpha(\gamma+H)=\gamma+\Gamma \cap H$ is a topoplogical isomorphism. Again since $\Gamma$ is open, $\varphi(\Gamma)=\Gamma+H / H$ is open in $\hat{G} / H$. By Theorem 1(ii), $\varphi(E) \cap(\Gamma+H / H) \subseteq \varphi(E)$ is a small $p$ set in $\hat{G} / H$, and thus since $\Gamma+H / H$ is open, it is also small $p$ in $\Gamma+H / H$. Topological isomorphisms preserve small $p$ sets, hence $\alpha(\varphi(E) \cap(\Gamma+H / H))$ is small $p$ in $\Gamma / \Gamma \cap H$. It is not difficult to check that $\varphi_{0}(E \cap \Gamma) \subseteq$ $\alpha(\varphi(E) \cap(\Gamma+H / H))$, and consequently we find that $\varphi_{0}(E \cap \Gamma)$ is a small $p$ set in $\Gamma / \Gamma \cap H$, i.e., (ii)' holds.

Finally, suppose $\overline{\rho_{0}\left(G / \Gamma^{\perp}\right)}$ is metrizable in $G_{1} / \Gamma_{1}^{\perp}$. Since $\Gamma$ is $\sigma$-compact, the quotient $G / \Gamma^{\perp}$ is metrizable. Thus, in light of (i)' and (ii)' we may apply Theorem 1 to $G / \Gamma^{\perp}, G_{1} / \Gamma_{1}^{\perp}$, and $\rho_{0}$ to conclude that $S \cap \Gamma$ is small $p q$ in $\Gamma$. However, $\Gamma$ is open in $\hat{G}$, from which it follows that $S \cap \Gamma$ is a small $p q$ set in $\hat{G}$, and the proof is complete. 
DEFINITION 2. We shall call a continuous homomorphism $\rho: G \rightarrow G_{1}$ between LCA groups $G$ and $G_{1}$ a topological homomorphism if $\rho$ is an open mapping of $G$ onto $\rho(G)$, i.e., if $\rho(V)$ is relatively open in $\rho(G) \subseteq G_{1}$ for each open subset $V \subseteq G$. Note that under such a map the subgroup $\rho(G)$ is locally compact and therefore closed in $G_{1}$.

The use of the measure $\omega \in M_{0}(G)$ in the proof of the next theorem is patterned after [12], where the original idea is attributed to S. Saeki.

THEOREM 2. Let $G$ be an LCA group and let $S$ and $E$ be measurable subsets of $\hat{G}$ such that $S \subseteq E$. Suppose $G_{1}$ is an $L C A$ group and $\rho: G \rightarrow G_{1}$ is a continuous homomorphism such that

(i) $\bar{\rho}^{-1}(\gamma+S)$ is a small $q$ set in $\hat{G}_{1}$ for a dense set of $\gamma \in \hat{G}$, and

(ii) $\varphi(E)$ is a small $p$ set in $\hat{G} /(\operatorname{ker} \rho)^{\perp}$. If either

(iii) $\overline{\rho(G)}$ is metrizable, or

(iv) $\rho$ is a topological homomorphism, then $S$ is a small pq set in $\hat{G}$.

Proof. Let $r=p q$ and suppose to the contrary that $S$ is not a small $r$ set in $\hat{G}$. Let $\mu \in M(G)$ be such that $\hat{\mu}=0$ off $S$, yet $\mu^{r} \notin L^{1}(G)$. Then by ([8, Corollary 3]) there exists a measure $\omega \in M_{0}^{+}(G)$ such that $\mu^{r} * \omega^{r} \notin$ $L^{1}(G)$.

Since $\omega \in M_{0}(G)$, supp $\hat{\omega}$ is $\sigma$-compact and hence there exists an open $\sigma$-compact subgroup $\Gamma$ in $\hat{G}$ containing supp $\hat{\omega}$. Let $\rho_{0}: G / \Gamma^{\perp} \rightarrow G_{1} / \Gamma_{1}^{\perp}$ be the induced homomorphism as in Lemma 2. Now either of the conditions (iii) or (iv) implies $\overline{\rho_{0}\left(G / \Gamma^{\perp}\right)}$ is metrizable, as we shall show shortly. Assuming this to be true for the moment, we complete the proof as follows. Since (i) and (ii) hold, Lemma 2 applies to show that $S \cap \Gamma$ is small $r$ in $\hat{G}$. Then since $(\mu * \omega)$ vanishes off $S \cap \Gamma$, we have $\mu^{r} * \omega^{r}=$ $(\mu * \omega)^{r} \in L^{1}(G)$, a contradiction. Thus, $S$ is a small $r$ set in $\hat{G}$.

It remains to show that either (iii) or (iv) implies that $\overline{\rho_{0}\left(G / \Gamma^{\perp}\right)}$ is metrizable. Let $\pi: G \rightarrow G / \Gamma^{\perp}$ and $\pi_{1}: G_{1} \rightarrow G_{1} / \Gamma_{1}^{\perp}$ be the natural quotient maps, so that $\rho_{0} \circ \pi=\pi_{1} \circ \rho$. Assume (iii) holds. Then since $\Gamma_{1}^{\perp}=$

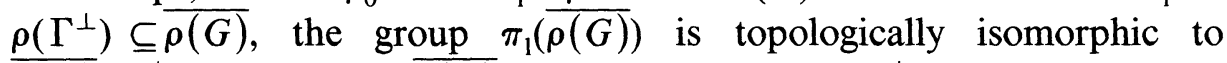
$\overline{\rho(G)} / \Gamma_{1}^{\perp}$, and thus $\pi_{1}(\overline{\rho(G)})$ is metrizable. Now $\Gamma_{1}^{\perp}$ is compact $\left(\Gamma_{1}=\right.$ $\bar{\rho}^{-1}(\Gamma)$ is open) so that $\pi_{1}$ is a closed map, and therefore since $\underline{\rho_{0}\left(G / \Gamma^{\perp}\right) \subseteq}$ $\pi_{1}(\overline{\rho(G)})$, we conclude that $\overline{\rho_{0}\left(G / \Gamma^{\perp}\right)} \subseteq \pi_{1}(\overline{\rho(G)})$. Hence, $\overline{\rho_{0}\left(G / \Gamma^{\perp}\right)}$ is metrizable. On the other hand, if (iv) holds, then it follows that the induced map $\rho_{0}: G / \Gamma^{\perp} \rightarrow G_{1} / \Gamma_{1}^{\perp}$ is also a topological homomorphism. Since $G / \Gamma^{\perp}$ is metrizable ( $\Gamma$ is $\sigma$-compact), we find once again that $\overline{\rho_{0}\left(G / \Gamma^{\perp}\right)}=\rho_{0}\left(G / \Gamma^{\perp}\right)=\left(G / \Gamma^{\perp}\right) / \operatorname{ker} \rho_{0}$ is metrizable. 
REMARKs. 1. At least one of the conditions (iii) or (iv) of Theorem 2 is satisfied in the following situations: $G$ arbitrary and $G_{1}$ metrizable, or $G_{1}$ arbitrary and $G \sigma$-compact with $\rho(G)$ closed (in particular, if $G$ is compact).

It is perhaps worth pointing out that the metrizability requirements arise solely as a result of the disintegration process, i.e., the requirement that $\overline{\rho(G)}$ be metrizable is used essentially at one point only - in the proof of Theorem 1 to guarantee the existence of a disintegration. If one could disintegrate $\mu$ with respect to $\rho: G \rightarrow G_{1}$ for general LCA $G_{1}$, then the above proofs would carry over without restriction to yield Theorem 2 for arbitrary $G, G_{1}$, and $\rho$. Although we have neither a reference nor a proof, it is nevertheless tempting to conjecture that this is true.

2. If $\hat{\mu}$ vanishes off $S$, and $\mu$ is a positive measure, or, more generally, if there exists a sequence $\left(Q_{n}\right)$ of trigonometric polynomials such that $Q_{n} \mu^{q} \rightarrow|\mu|^{q}$ are (equivalently, $\left|\mu^{q}\right|$ and $|\mu|^{q}$ are absolutely continuous with respect to one another), then in fact $\mu^{\max (p, q)} \in L^{1}(G)$. This may be seen by modifying the proof of Theorem 1 as follows. Set $\nu=\rho(|\mu|)$, rather than $\nu=\rho\left(\left|\mu^{q}\right|\right)$, and argue as in the beginning of the proof of Theorem 1 to show that $\nu^{q} \in L^{\mathrm{l}}\left(G_{1}\right)$. Then disintegrate $\mu$ to obtain formula (4) with $\mu^{q}$ replaced by $\mu$. The middle portion of the proof is the same as before up to line (14). This is now modified to read

$$
\omega(f)=\int_{G_{1}^{r}} \lambda_{y_{1}} * \cdots * \lambda_{y_{r}}(f) d(\nu \times \cdots \times \nu)\left(y_{1}, \ldots, y_{r}\right),
$$

where $r=\max (p, q)$. Then only minor changes are necessary in the tail end of the proof to conclude that $\mu^{r} \in L^{1}(G)$. These same conclusions hold for Theorem 2 .

We do not know if $S$ is, in general, a small $\max (p, q)$ set in $\hat{G}$. If condition (i) is altered to read

(i) $\bar{\rho}^{-1}\left(\left\{\gamma_{1}, \ldots, \gamma_{q}\right\}+S\right)$ is a small $q$ set in $\hat{G}_{1}$ whenever $\gamma_{1}, \ldots, \gamma_{q} \in D$ ( $D$ dense in $\hat{G}$ ), then one can show that $S$ is a small $\max (p, q)$ set. In this case, $\rho(Q \mu)^{q} \in L^{1}\left(G_{1}\right)$ for each trigonometric polynomial $Q$, and it follows that $\nu^{q} \in L^{1}\left(G_{1}\right)$, where $\nu=\rho(|\mu|)$. We may then proceed as outlined in the last paragraph.

THEOREM 3. Let $G$ be an LCA group and $S$ a measurable subset of $\hat{G}$. In order that $S$ be a small $r$ set in $\hat{G}$ it is necessary and sufficient that there exist a closed subgroup $\Gamma \subseteq \hat{G}$ and positive integers $p$ and $q$ such that

(i) $r=p q$,

(ii) $(\gamma+S) \cap \Gamma$ is a small $q$ set in $\Gamma$ for a dense set of $\gamma \in G$, and

(iii) $\varphi(S)$ is a small $p$ set in $\hat{G} / \Gamma$. 
Proof. If $S$ is a small $r$ set in $\hat{G}$ let $q=r, p=1$, and $\Gamma=\hat{G}$. Then (i) and (iii) clearly hold, and (ii) holds since the translate of a small $r$ set is again a small $r$ set.

Conversely, assume (i)-(iii) hold. Let $\rho: G \rightarrow G / \Gamma^{\perp}$ be the standard quotient map, so that the adjoint $\bar{\rho}: \Gamma \rightarrow \hat{G}$ is the injection of $\Gamma$ into $\hat{G}$. Since $\rho$ is an open map and (i) and (ii) of Theorem 2 hold (with $S=E$ ) by virtue of (ii) and (iii) above, Theorem 2 applies to show that $S$ is a small $r=p q$ set in $\hat{G}$.

To illustrate Theorem 3, we use the F. and M. Riesz Theorem on $Z$ to derive its analogue in $R$, that $R^{+}=[0, \infty)$ is a Riesz set in $R([\mathbf{1 0}, 8.2 .7])$. Take $S=R^{+}, G=R$, and $\Gamma=Z$ in Theorem 3 with $p=q=1$. Then (iii) holds since $R / Z$ is compact, and (ii) holds since $\left(x+R^{+}\right) \cap Z$ is a translate in $Z$ of $Z^{+}$for each $x \in R$ and is therefore a Riesz set in $Z$ by the F. and M. Riesz theorem.

Now let $h: \hat{G} \rightarrow \hat{G}_{0}$ be a continuous homomorphism and $T$ a small $p$ set in $\hat{G}_{0}$. For $S \subseteq h^{-1}(T)$ we seek conditions from which we may infer that $S$ is a small $q$ set in $\hat{G}$ for some $q \geq 1$. Some additional conditions are required, inverse images of small $p$ sets behave poorly even under very reasonable circumstances. Consider, for example, the imbedding $h: Z \rightarrow R$ of $Z$ into $R$. Here every subset $S$ of $Z$ is a Riesz set in $R$, but, of course, $h^{-1}(S)=S$ need not be a Riesz (or small $q$ ) set in $Z$, even though $h$ is a 1-1 topological homomorphism into $R$. Again, if $\hat{G}$ is not compact and $h$ : $\hat{G} \rightarrow \hat{G}_{b}$ is the injection of $\hat{G}$ into its Bohr compactification $\hat{G}_{b}$, then every measurable subset $T \subseteq \hat{G}_{b}$ is Riesz in $\hat{G}_{b}$, but $h^{-1}(T)=T \cap \hat{G}$ need not be small $q$ in $\hat{G}$ for any $q \geq 1$.

If, in Theorem 2, we take $E=h^{-1}(T)$ and suppose (i) and (ii) hold ( $\rho$ as in (iii) or (iv)), then we force $S$ to be small $p q$ set in $\hat{G}$. This is the idea behind the next theorem and its corollaries and, thus, also behind the applications which follow.

TheOREM 4. Let $G, G_{1}$, and $G_{2}$ be LCA groups and consider the mappings

$$
\hat{G}_{1} \stackrel{g}{\rightarrow} \hat{G} \stackrel{h}{\rightarrow} \hat{G}_{2}
$$

where $h$ is a topological homomorphism, and $g$ is either a topological homomorphism or a continuous homomorphism such that $\hat{G}_{1} / \mathrm{ker} g$ is $\sigma$-compact. Let $\Gamma=\operatorname{ker} h$ and $\Lambda=\overline{g\left(\hat{G}_{1}\right)}$. Assume $h(\Lambda)$ is closed in $\hat{G}_{2}$, and let $\varphi_{1}: h(\hat{G}) \rightarrow h(\hat{G}) / h(\Lambda)$ be the natural quotient map. Suppose $T$ is a small $p$ set in $h(\hat{G}) / h(\Lambda)$ and $S \subseteq\left(\varphi_{1} \circ h\right)^{-1}(T)$ is measurable. If

(i) $g^{-1}(\gamma+S)$ is a small $q$ set in $\hat{G}_{1}$ for a dense set of $\gamma \in \hat{G}$, and 
(ii) $\left[\chi+\Lambda+\varphi\left(\left(\varphi_{1} \circ h\right)^{-1}(T)\right)\right] \cap(\Gamma+\Lambda / \Lambda)$ is a small $r$ set in $\Gamma+\Lambda / \Lambda$ for a dense set of $\chi+\Lambda \in \hat{G} / \Lambda$, then $S$ is a small pqr set in $\hat{G}$. In particular, if

(iii) $\Gamma+\Lambda / \Lambda$ is compact and (i) holds, then $S$ is a small pq set in $\hat{G}$. The group $\Gamma+\Lambda / \Lambda$ is compact if either $\Gamma$ is compact, or $\hat{G} / \Lambda$ is compact, or $\Gamma \subseteq \Lambda$, and so in these cases (i) alone is sufficient to guarantee that $S$ is a small pq set in $\hat{G}$.

Proof. We first note that since $h(\Lambda)$ is assumed to be closed in $\hat{G}_{2}$, $\Gamma+\Lambda=h^{-1}(h(\Lambda))$ is closed in $\hat{G}$, and thus the group $\Gamma+\Lambda / \Lambda$ appearing in (ii) is an LCA group.

If we denote the adjoint of $g$ by $\rho: G \rightarrow G_{1}$ (hence $\bar{\rho}=g$ ), then $\rho$ satisfies either (iii) or (iv) of Theorem 2. For if $g$ is a topological homomorphism, then so is $\rho$, and if $\hat{G}_{1} / \operatorname{ker} g$ is $\sigma$-compact, then $\overline{\rho(G)}=$ $(\operatorname{ker} g)^{\perp}$ is metrizable. Moreover, since $g\left(\hat{G}_{1}\right)$ is dense in $(\operatorname{ker} \rho)^{\perp}$, we have $\Lambda=(\operatorname{ker} \rho)^{\perp}$. Thus, by (i) and Theorem 2 it suffices to show that $\varphi\left(\left(\varphi_{1} \circ h\right)^{-1}(T)\right)$ is a small $p r$ set in $\hat{G} / \Lambda$.

Let $\alpha: \hat{G} / \Lambda \rightarrow h(\hat{G}) / h(\Lambda) ; \alpha(\gamma+\Lambda)=h(\gamma)+h(\Lambda)$ be the continuous homomorphism induced by $h$, and let

$$
\hat{G} / \Lambda \stackrel{\pi}{\rightarrow}(\hat{G} / \Lambda) / \operatorname{ker} \alpha \stackrel{\psi}{\rightarrow} h(\hat{G}) / h(\Lambda)
$$

be the canonical factorization of $\alpha$. Since $h$ is a topological homomorphism, $\alpha$ is an open surjection and, consequently, $\psi$ is a topological isomorphism. By hypothesis, $T$ is small $p$ in $h(\hat{G}) / h(\Lambda)$, and thus $\psi^{-1}(T)$ is small $p$ in $(\hat{G} / \Lambda) / \operatorname{ker} \alpha$. We have $\pi\left(\varphi\left(\left(\varphi_{1} \circ h\right)^{-1}(T)\right)\right)=\psi^{-1}(T)$, so $\pi\left(\varphi\left(\left(\varphi_{1} \circ h\right)^{-1}(T)\right)\right)$ is a small $p$ set in $(\hat{G} / \Lambda) / \operatorname{ker} \alpha$. Now $\operatorname{ker} \alpha=$ $\varphi(\operatorname{ker} h)=\Gamma+\Lambda / \Lambda$, and this combined with (ii) shows that $[\chi+\Lambda+$ $\left.\varphi\left(\left(\varphi_{1} \circ h\right)^{-1}(T)\right)\right] \cap \operatorname{ker} \alpha$ is small $r$ in ker $\alpha$ for a dense set of $\chi+\Lambda \in$ $\hat{G} / \Lambda$. Therefore, by Theorem $3, \varphi\left(\left(\varphi_{1} \circ h\right)^{-1}(T)\right)$ is a small pr set in $\hat{G} / \Lambda$, which is what we wished to show.

Lastly, if $\Gamma+\Lambda / \Lambda$ is compact, then (ii) holds with $r=1$ so that $S$ is a small $p q$ set in $\hat{G}$. If $\Gamma$ is compact, then $\varphi(\Gamma)=\Gamma+\Lambda / \Lambda$ is also, and if $\hat{G} / \Lambda$ is compact, then the closed subgroup $\Gamma+\Lambda / \Lambda$ is again compact. If $\Gamma \subseteq \Lambda$, then $\Gamma+\Lambda / \Lambda=\{0\}$ is clearly compact, and the proof is complete.

Several results akin to Theorem 4 may be obtained by adapting the proof suitably. We state one such variant below. Its proof is a direct consequence of Theorem 3 and the following observations. If $h: \hat{G} \rightarrow \hat{G}_{0}$ is a topological homomorphism, $\Gamma$ a closed subgroup of $h(\hat{G})$, and $T \subseteq$ $h(\hat{G}) / \Gamma$, then the induced homomorphism $\alpha: \hat{G} / h^{-1}(\Gamma) \rightarrow h(\hat{G}) / \Gamma$ is a 
topological isomorphism and $\alpha^{-1}(T)=\left(\varphi\left(\varphi_{1} \circ h\right)^{-1}(T)\right)$, where $\varphi: \hat{G} \rightarrow$ $\hat{G} / h^{-1}(\Gamma)$ and $\varphi_{1}: h(\hat{G}) \rightarrow h(\hat{G}) / \Gamma$ denote the associated quotient maps.

THEOREM 5. Let $G$ and $G_{0}$ be LCA groups and let $h: \hat{G} \rightarrow \hat{G}_{0}$ be a topological homomorphism. Let $\Gamma$ be a closed subgroup of $h(\hat{G})$ and denote the quotient map $h(\hat{G}) \rightarrow h(\hat{G}) / \Gamma$ by $\varphi_{1}$. Suppose $T$ is a small $p$ set in $h(\hat{G}) / \Gamma$ and $S \subseteq\left(\varphi_{1} \circ h\right)^{-1}(T)$ is measurable. If

(i) $(\gamma+S) \cap h^{-1}(\Gamma)$ is a small $q$ set in $h^{-1}(\Gamma)$ for a dense set of $\gamma \in \hat{G}$, then $S$ is a small pq set in $\hat{G}$.

REMARK. It is perhaps worth pointing out that if $H$ is a closed subgroup of $\hat{G}$ and $S \subseteq H$ is a small $p$ set in $H$, then $S$ is also a small $p$ set in $\hat{G}$. This can be made a consequence of the above, but there is a more direct route. If $H$ is open, then $S$ is small $p$ in $\hat{G}$ by [11, Lemma 6]. If $H$ is not open, then it is locally null in $\hat{G}$ (otherwise $H$ contains a compact subset $K$ of positive measure, and thus $K-K$ contains a neighborhood of $0)$. Then $H^{c}$, and hence $S^{c}$ also, is dense in $\hat{G}$. Consequently, if $\hat{\mu}$ vanishes off $S$, then, by continuity, $\mu=0$ and so certainly $S$ is small $p$ in $\hat{G}$. The converse is true if $H$ is open (again by [11]), but is false generally; if $H$ is a closed subgroup of $\hat{G}$ which is not open, and if $S$ is small $p$ in $\hat{G}$, then $S \cap H$ need not be small $q$ in $H$ for any $q \geq 1$ (e.g., $Z$ in $R$ ).

Corollary 1. Let $G, G_{1}$, and $G_{2}$ be LCA groups and consider the mappings

$$
\hat{G}_{1} \stackrel{g}{\rightarrow} \hat{G} \stackrel{h}{\rightarrow} \hat{G}_{2}
$$

where $h$ is a topological homomorphism, and $g$ is either a topological homomorphism or a continuous homomorphism such that $\hat{G}_{1} / \mathrm{ker} g$ is $\sigma$-compact. Assume $g\left(\hat{G}_{1}\right) \subseteq \operatorname{ker} h$. Suppose $T$ is a small $p$ set in $h(\hat{G})$ and $S \subseteq h^{-1}(T)$ is measurable. If

(i) $g^{-1}(\gamma+S)$ is a small $q$ set in $\hat{G}_{1}$ for a dense set of $\gamma \in \hat{G}$, and

(ii) $\operatorname{ker} h / \overline{g\left(\hat{G}_{1}\right)}$ is compact, then $S$ is a small pq set in $\hat{G}$.

In particular, if $g\left(\hat{G}_{1}\right)=\operatorname{ker} h$, or if $\operatorname{ker} h$ is compact, then (i) alone implies $S$ is a small pq set in $\hat{G}$.

Proof. Let $\Gamma=\operatorname{ker} h$ and $\Lambda=\overline{g\left(\hat{G}_{1}\right)}$ as in Theorem 4. By hypothesis $\Lambda \subseteq \Gamma$, whence $h(\Lambda)=\{0\}$, so we may clearly suppose, in Theorem 4, that $T \subseteq h(\hat{G})$ and $S \subseteq h^{-1}(T)$. By (ii), $\Gamma+\Lambda / \Lambda=\Gamma / \Lambda$ is compact, and this, together with (i) and Theorem 4(iii), shows that $S$ is small $p q$ in $\hat{G}$. 
Corollary 2. Let $G$ and $G_{0}$ be LCA groups, $h: \hat{G} \rightarrow \hat{G}_{0}$ topological homomorphism, and $\Lambda$ a closed subgroup of $\hat{G}$. Assume $h(\Lambda)$ is closed in $\hat{G}_{0}$ and let $\varphi_{1}: h(\hat{G}) \rightarrow h(\hat{G}) / h(\Lambda)$ be the natural quotient map. Suppose $T$ is a small p set in $h(\hat{G}) / h(\Lambda)$ and $S \subseteq\left(\varphi_{1} \circ h\right)^{-1}(T)$ is measurable. If

(i) $(\gamma+S) \cap \Lambda$ is a small $q$ set in $\Lambda$ for a dense set of $\gamma \in \hat{G}$, and

(ii) either $\operatorname{ker} h$ is compact, or $\hat{G} / \Lambda$ is compact, or $\operatorname{ker} h \subseteq \Lambda$, then $S$ is a small pq set in $\hat{G}$.

Furthermore, if

(iii) $(\gamma+S) \cap(\Lambda+\operatorname{ker} h)$ is a small $q$ set in $\Lambda+\operatorname{ker} h$ for a dense set of $\gamma \in \hat{G}$,

then $S$ is a small pq set in $\hat{G}$. In particular, if $\Lambda+\operatorname{ker} h$ is compact, then $\left(\varphi_{1} \circ h\right)^{-1}(T)$ is a small $p$ set in $\hat{G}$.

Proof. For the first part apply Theorem 4 (iii) with $\hat{G}_{1}=\Lambda$ and $g$ equal to the injection of $\Lambda$ into $\hat{G}$. For the second part apply Theorem 5 with $\Gamma$ there equal to $h(\Lambda)$ and observe that $h^{-1}(h(\Lambda))=\Lambda+\operatorname{ker} h$. If $\Lambda+\operatorname{ker} h$ is compact, then (iii) holds with $q=1$ and $S=\left(\varphi_{1} \circ h\right)^{-1}(T)$.

COROLlaRY 3. Let $G$ and $G_{0}$ be LCA groups.

(1) Let $h: \hat{G} \rightarrow \hat{G}_{0}$ be a topological homomorphism, $T$ a small $p$ set in $h(\hat{G})$, and $S \subseteq h^{-1}(T)$ a measurable subset. If

(i) $(\gamma+S) \cap \operatorname{ker} h$ is a small $q$ set in $\operatorname{ker} h$ for a dense set of $\gamma \in \hat{G}$, then $S$ is a small pq set in $\hat{G}$. In particular, if $T$ is a small p set in $h(\hat{G})$ and ker $h$ is compact, then $h^{-1}(T)$ is a small p set in $\hat{G}$.

(2)([11]). If $\Lambda$ is a compact subgroup of $\hat{G}$ and $T$ is a small pet in $\hat{G} / \Lambda$, then $\varphi^{-1}(T)$ is a small $p$ set in $\hat{G}$, where $\varphi: \hat{G} \rightarrow G / \Lambda$ is the natural quotient map.

Proof. For part (1) apply Corollary 2 (iii) with $\Lambda=\{0\}$. For part (2) apply the "in particular" statement in part (1) to the quotient map $\varphi$ : $\hat{G} \rightarrow \hat{G} / \Lambda$.

As we mentioned earlier, it is shown in [12] that if $S_{1} \subseteq \hat{G}_{1}$ is a Riesz set and $S_{2} \subseteq \hat{G}_{2}$ is a small $p$ set, then the cartesian product $S_{1} \times S_{2}$ is a small $p$ set in $\hat{G}_{1} \times \hat{G}_{2}$. This may be regarded as a generalization to LCA groups of Bochner's F. and M. Riesz theorem for $Z \times Z$. The following theorem provides a further extension along the same lines.

THEOREM 6. Let $G_{1}, \ldots, G_{n}$ be LCA groups. If $S_{j}$ is a small $p_{j}$ set in $\hat{G}_{j}$ for each $j=1, \ldots, n$, then the cartesian product $S_{1} \times \cdots \times S_{n}$ is a small $p_{1} p_{2} \cdots p_{n}$ set in $\hat{G}_{1} \times \cdots \times \hat{G}_{n}$. In particular, the cartesian product of Riesz sets is again a Riesz set. 
Proof. By induction it is sufficient to establish the theorem for $n=2$. We consider the mappings

$$
\hat{G}_{1} \stackrel{j_{1}}{\rightarrow} \hat{G}_{1} \times \hat{G}_{2} \stackrel{\pi_{2}}{\rightarrow} \hat{G}_{2}
$$

where $j_{1}(\gamma)=(\gamma, 0)$ is the injection of $\hat{G}_{1}$ into $\hat{G}_{1} \times \hat{G}_{2}$, and $\pi_{2}(\gamma, \chi)=\chi$ is the projection of $\hat{G}_{1} \times \hat{G}_{2}$ onto $\hat{G}_{2}$. The maps $j_{1}$ and $\pi_{2}$ are topological homomorphisms with $j_{1}\left(\hat{G}_{1}\right)=\hat{G}_{1} \times\{0\}=\operatorname{ker} \pi_{2}$. Thus, since $S_{2}$ is a small $p_{2}$ set in $\hat{G}_{2}$ and $S_{1} \times S_{2} \subseteq \hat{G}_{1} \times S_{2}=\pi_{2}^{-1}\left(S_{2}\right)$, we need only show, by Corollary 1, that $j_{1}^{-1}\left((\gamma, \chi)+S_{1} \times S_{2}\right)$ is a small $p_{1}$ set in $\hat{G}_{1}$ for a dense set of $(\gamma, \chi) \in \hat{G}_{1} \times \hat{G}_{2}$. But for each $(\gamma, \chi) \in \hat{G}_{1} \times \hat{G}_{2}$ we have

$$
j_{1}^{-1}\left((\gamma, \chi)+S_{1} \times S_{2}\right)= \begin{cases}\varnothing & \text { if } 0 \notin \chi+S_{2}, \\ \gamma+S_{1} & \text { if } 0 \in \chi+S_{2} .\end{cases}
$$

Thus, since, by hypothesis, $S_{1}$ is small $p_{1}$ in $\hat{G}_{1}$, Corollary 1 applies to show that $S_{1} \times S_{2}$ is small $p_{1} p_{2}$ in $\hat{G}_{1} \times \hat{G}_{2}$, which completes the proof.

By a proper cone in $R^{n}$ we shall mean a subset of the form

$$
C\left(v, x_{0}, r\right)=\bigcup_{x \in B\left(x_{0}, r\right)}\{(1-t) v+t x: t \geq 0\},
$$

where $B\left(x_{0}, r\right)$ is a closed ball in $R^{n}$ of radius $r>0$ with center at $x_{0}$, and $v$ is a point in $R^{n}$ not contained in $B\left(x_{0}, r\right)$. A proper cone in $R^{n}$ is closed and convex. Geometrically, $C\left(v, x_{0}, r\right)$ consists of all half-lines issuing from $v$ which pass through the ball $B\left(x_{0}, r\right)$. A proper cone in $Z^{n}$ is by definition a subset of $Z^{n}$ of the form $C\left(v, x_{0}, r\right) \cap Z^{n}$, where $C\left(v, x_{0}, r\right)$ is a proper cone in $R^{n}$ with $v$ and $x_{0}$ in $Z^{n}$.

Note that a proper cone in $Z$ (or $R$ ) is simply a half-line and is therefore, by the F. and M. Riesz theorem, a Riesz set. The classical Bochner theorem ([1, Theorem 5]) is the higher-dimensional analogue of the F. and M. Riesz theorem and may be accurately paraphrased by stating that any proper cone in $Z^{n}$ is a Riesz set. By combining the earlier material and the F. and M. Riesz theorem we obtain the following extension of Bochner's theorem.

By a hyperplane $H$ in $R^{n}$ we mean an ( $\left.n-1\right)$-dimesional subspace of $R^{n}$. Denoting the usual inner product of $x$ and $y$ in $R^{n}$ by $x \cdot y$, we thus have $H=\left\{x \in R^{n}: x \cdot a=0\right\}$ for some $a \in R^{n}, a \neq 0$. The sets $H_{1}=\{x$ $\left.\in R^{n}: x \cdot a \geq 0\right\}$ and $H_{2}=\left\{x \in R^{n}: x \cdot a \leq 0\right\}$ will be called the closed half spaces determined by $H$. We define a hyperplane in $Z^{n}$ to be a subgroup of the form $H=\left\{x \in Z^{n}: x \cdot a=0\right\}$, where $a \in Z^{n}, a \neq 0$. The corresponding sets $H_{1}=\left\{x \in Z^{n}: x \cdot a \geq 0\right\}$ and $H_{2}=\left\{x \in Z^{n}\right.$ : $x \cdot a \leq 0\}$ will again be called the closed half spaces determined by $H$. 
THEOREM 7. Let $S$ be a measurable subset of $R^{n}$ [any subset of $Z^{n}$ ] such that a translate of $S$ lies in a closed half space determined by some hyperplane $H$ in $R^{n}\left[Z^{n}\right]$. If $(x+S) \cap H$ is a small $p$ set in $H$ for a dense set of $x \in R^{n}\left[Z^{n}\right]$, then $S$ is a small $p$ set in $R^{n}\left[Z^{n}\right]$. In particular, if $(x+S) \cap H$ has finite measure in $H$ for a dense set of $x \in R^{n}\left[Z^{n}\right]$, then $S$ is a Riesz set in $R^{n}\left[Z^{n}\right]$.

Proof. Since small $p$ sets are preserved by translation we may assume that $S$ itself lies in one of the half spaces determined by $H$, say, $H_{1}=\{x$ : $x \cdot a \geq 0\}$.

Consider the case of $R^{n}$ first. The linear map $R^{n} \rightarrow R ; h(x)=x \cdot a$ is a topological homomorphism of $R^{n}$ onto $R\left(a \in R^{n}, a \neq 0\right)$ with $\operatorname{ker} h=$ $H$. The set $T=\{x \in R: x \geq 0\}$ is a Riesz set in $R$ and, by hypothesis, $S \subseteq H_{1}=h^{-1}(T)$. Since $(x+S) \cap \operatorname{ker} h$ is a small $p$ set in $\operatorname{ker} h$ for a dense set of $x \in R^{n}$, Corollary 3(1) applies, showing that $S$ is a small $p$ set in $R^{n}$.

The proof for $Z^{n}$ is similar. The map $h: Z^{n} \rightarrow Z$ defined by $h(x)=$ $x \cdot a$ is a topological homomorphism of $Z^{n}$ onto a nontrivial subgroup of $Z\left(a \in Z^{n}, a \neq 0\right)$ with $\operatorname{ker} h=H$. Let $h\left(Z^{n}\right)=b Z$, where $b \in Z, b \geq 1$. Since $b Z$ is isomorphic to $Z$, the set $T=\{b n: n \geq 0\}$ is a Riesz set in $b Z$ and, as before, $S \subseteq H_{1}=h^{-1}(T)$. By hypothesis, $(x+S) \cap \operatorname{ker} h$ is a small $p$ set in $\operatorname{ker} h$ for all $x \in Z^{n}$. Thus, again by Corollary 3(1), $S$ is small $p$ in $Z^{n}$, and the proof is complete.

Bochner's theorem, that a proper cone is a Riesz set, is contained in Theorem 7. This is evident on purely geometric grounds, since if $C$ is a proper cone it seems clear that we may choose a hyperplane $H$ such that any translate of $C$ intersects $H$ in a set of finite measure. A proof follows.

THEOREM 8 [Bochner]. Any proper cone in $Z^{n}$ or $R^{n}$ is a Riesz set.

Proof. Assume first that $C$ is a proper cone in $R^{n}$. The translate of a proper cone in $R^{n}$ is again a proper cone so we may suppose the vertex of $C$ is $v=0$, i.e., we may assume $C=C\left(0, x_{0}, r\right)$, where $x_{0} \in R^{n}, x_{0} \neq 0$, and $r>0$. Let $H$ be the hyperplane

$$
H=\left\{x \in R^{n}: x \cdot x_{0}=0\right\},
$$

i.e., $H$ is the orthogonal complement of the 1-dimensional subspace $\left\{t x_{0}\right.$ : $t \in R\}$. We will show that $C$ is contained in one of the half spaces determined by $H$ and that $(x+C) \cap H$ has finite measure in $H$ for every $x \in R^{n}$. It will then follow from Theorem 7 that $C$ is a Riesz set in $R^{n}$. 
These assertions are a consequence of the following claim.

Claim: for each $z_{0} \in R^{n}$ and $w \in\left(z_{0}+C\right) \cap H$ we have

$$
\|w\| \leq\left\|z_{0}\right\|+\delta^{-1}\left|z_{0} \cdot x_{0}\right|\left(r+\left\|x_{0}\right\|\right),
$$

where $\delta>0$ is a constant depending only on $C$.

The claim is established as follows. The function $f(x)=\left|x \cdot x_{0}\right|$ is continuous, and thus $f\left(B\left(x_{0}, r\right)\right)=\left\{\left|x \cdot x_{0}\right|: x \in B\left(x_{0}, r\right)\right\}$ is compact. Suppose $0 \in f\left(B\left(x_{0}, r\right)\right)$. Then $x \cdot x_{0}=0$ for some $x \in B\left(x_{0}, r\right)$. But $0 \notin B\left(x_{0}, r\right)$ since $C$ is a proper cone with $v=0$ and, consequently, $\left\|x_{0}\right\|>r$. Then

$$
\|x\|^{2}+r^{2}<\|x\|^{2}+\left\|x_{0}\right\|^{2}=\left\|x-x_{0}\right\|^{2} \leq r^{2},
$$

the middle equality holding since $x \cdot x_{0}=0$. This contradiction shows $0 \notin f\left(B\left(x_{0}, r\right)\right)$ and, therefore, $\delta=\operatorname{dist}\left(0, f\left(B\left(x_{0}, r\right)\right)\right)>0$. In particular, $\left|x \cdot x_{0}\right| \geq \delta>0$ for all $x \in B\left(x_{0}, r\right)$.

Now, let $w \in\left(z_{0}+C\right) \cap H$. Since $w-z_{0} \in C$, there exists an $x \in$ $B\left(x_{0}, r\right)$ and a $t \geq 0$ such that $w-z_{0}=t x$. Since also $w \in H$, we have

$$
0=\left|w \cdot x_{0}\right|=\left|\left(t x+z_{0}\right) \cdot x_{0}\right| \geq t\left|x \cdot x_{0}\right|-\left|z_{0} \cdot x_{0}\right| \geq t \delta-\left|z_{0} \cdot x_{0}\right|,
$$

i.e., $t \leq \delta^{-1}\left|z_{0} \cdot x_{0}\right|$. Then

$$
\|w\| \leq\left\|z_{0}\right\|+t\|x\| \leq\left\|z_{0}\right\|+\delta^{-1}\left|z_{0} \cdot x_{0}\right|\left(r+\left\|x_{0}\right\|\right)
$$

as claimed.

Setting $z_{0}=0$ in (1) we find that $C \cap H=\{0\}$. Since $C \backslash\{0\}$ is connected, it follows that $C$ lies entirely in one of the half spaces determined by $H$. On the other hand, if $z_{0} \in R^{n}$, then $\left(z_{0}+C\right) \cap H$ is closed and is also bounded by (1), hence compact. Consequently, $\left(z_{0}+\right.$ $C) \cap H$ has finite measure in $H$ for every $z_{0} \in R^{n}$, so $C$ is a Riesz set in $R^{n}$.

Consider next the proper cone $C=C\left(v, x_{0}, r\right) \cap Z^{n}, v, x_{0} \in Z^{n}$, in $Z^{n}$. As before we may assume $v=0$ (and thus $x_{0} \notin 0$ ). Let $H=\left\{x \in R^{n}\right.$ : $\left.x \cdot x_{0}=0\right\}$ be the hyperplane in $R^{n}$ generated by $x_{0}$. Then since $x_{0} \in$ $Z^{n} \backslash\{0\}, H \cap Z^{n}$ is a hyperplane in $Z^{n}$. We know from above that $C\left(0, x_{0}, r\right)$ lies in one of the half spaces determined by $H$, so, clearly, $C=C\left(0, x_{0}, r\right) \cap Z^{n}$ lies in one of the half spaces determined by $H \cap Z^{n}$. Moreover, for each $z_{0} \in Z^{n}$ we have

$$
\left(z_{0}+C\left(0, x_{0}, r\right) \cap Z^{n}\right) \cap H \cap Z^{n} \subseteq\left(z_{0}+C\left(0, x_{0}, r\right)\right) \cap H .
$$

Since $\left(z_{0}+C\left(0, x_{0}, r\right)\right) \cap H$ is compact, the set $\left(z_{0}+C\right) \cap H \cap Z^{n}$ is finite in $H \cap Z^{n}$. Thus, again by Theorem 7,C is a Riesz set in $Z^{n}$, and the proof is complete. 
Finally, let us call a subset $S$ in an LCA group $\hat{G}$ a conical Riesz set if there exists an open subgroup $\Gamma$ of $\hat{G}$ which contains $S$ and a topological isomorphism $\rho: R^{n} \times Z^{m} \times F \rightarrow \Gamma$ onto $\Gamma$ such that $S=\rho\left(C_{1} \times C_{2} \times F\right)$, where $C_{1}$ is a proper cone in $R^{n}(n \geq 0), C_{2}$ is a proper cone in $Z^{m}$ ( $m \geq 0$ ), and $F$ is a compact abelian group. It follows from Theorems 6 and 8 that such a set $S$ is, in fact, a Riesz set in $\hat{G}$. For $C_{1}$ and $C_{2}$ are Riesz sets in $R^{n}$ and $Z^{m}$ by Theorem 8 , and $F$ is clearly a Riesz set in itself; hence, by Theorem $6, C_{1} \times C_{2} \times F$ is a Riesz set in $R^{n} \times Z^{m} \times F$. Since $\tau$ is a topological isomorphism, $S=\rho\left(C_{1} \times C_{2} \times F\right)$ is a Riesz set in $\Gamma$ and also in $\hat{G}$ since $\Gamma$ is open.

Now, if $V$ is an open relatively compact (i.e., $\bar{V}$ is compact) neighborhood of 0 in $\hat{G}$, then $\operatorname{gp}(V)$, the group generated by $V$, is an open compactly generated subgroup of $\hat{G}$. Then, as is well $\operatorname{known}, \operatorname{gp}(V)$ is topologically isomorphic to $R^{n} \times Z^{m} \times F$ for some nonnegative integers $n$ and $m$, and some compact abelian group $F$. Combining these facts with the definition above we obtain the following version of Bochner's theorem.

THEOREM 9. Let $\hat{G}$ be an LCA group. Then every open compactly generated subgroup of $\hat{G}$ contains a conical Riesz set. In particular, for each open relatively compact neighborhood $V$ of 0 in $\hat{G}, g p(V)$ contains a conical Riesz set.

If $\hat{G}$ itself is compactly generated, say, $\hat{G}=R^{n} \times Z^{m} \times F$, where $n, m \geq 0$ and $F$ is a compact abelian group, then every subset of the form $C_{1} \times C_{2} \times F$, where $C_{1}$ is a proper cone in $R^{n}$ and $C_{2}$ is a proper cone in $Z^{m}$, is a Riesz set in $\hat{G}$.

\section{REFERENCES}

[1] S. Bochner, Boundary values of analytic functions in several variables and of almost periodic functions, Ann. Math., 45 (1944), 708-722.

[2] R. Dressler, W. Parker, and L. Pigno, Sidon sets and small p sets, Quart. J. Math. Oxford II. Ser., 24 (1973), 79-80.

[3] I. Glicksberg, Fourier-Stieltjes transforms with small supports, Illinois J. Math., 9 (1965), 418-426.

[4] C. C. Graham, Fourier-Stieltjes transforms with small supports, Illinois J. Math., 18 (1974), 532-534.

[5] E. Hewitt and K. A. Ross, Abstract Harmonic Analysis, Vol. I and II, Springer-Verlag, New York-Heidelberg-Berlin, 1963 and 1970.

[6] A. Ionescu Tulcea and C. Ionescu Tulcea, Topics in the Theory of Lifting, SpringerVerlag, New York, 1969.

[7] L. Pigno, Convolution products with small Fourier-Stieltjes transforms, Illinois J. Math., 19 (1975), 77-78. 
[8] L. Pigno and S. Saeki, Fourier-Stieltjes transforms which vanish at infinity, Math. Z., 141 (1975), 83-91.

[9] F. Riesz and M. Riesz, Uber Randwerte einer analytischen Funktion, Quatrieme Congres des Math. Scand., (1916), 27-44.

[10] W. Rudin, Fourier Analysis on Groups, Interscience Tract No. 12, Wiley, New York, 1962.

[11] H. Yamaguchi, Remarks on Riesz sets, Hokkaido Math. J., 7 (1978), 328-335.

[12] On the product of a Riesz set and a small p set, Proc. Amer. Math. Soc., 81 (1981), 273-278.

Received January 18, 1982.

Wichita STATE UNIVERSITY

WICHITA, KS 67208 



\section{PACIFIC JOURNAL OF MATHEMATICS \\ EDITORS}

Donald BABBITT (Managing Editor)

University of California

Los Angeles, CA 90024

Hugo Rossi

University of Utah

Salt Lake City, UT 84112

C. C. Moore and Arthur Ogus

University of California

Berkeley, CA 94720
J. DugunduI

Department of Mathematics

University of Southern California

Los Angeles, CA 90089-1113

R. FinN and H. SAMELSON

Stanford University

Stanford, CA 94305

ASSOCIATE EDITORS

R. ARENS

E. F. BECKENBACH

B. H. NeUMANN

F. WOLF

K. YosHIDA (1906-1982)

\section{SUPPORTING INSTITUTIONS}

UNIVERSITY OF ARIZONA

UNIVERSITY OF BRITISH COLUMBIA

CALIFORNIA INSTITUTE OF TECHNOLOGY

UNIVERSITY OF CALIFORNIA

MONTANA STATE UNIVERSITY

UNIVERSITY OF NEVADA, RENO

NEW MEXICO STATE UNIVERSITY

OREGON STATE UNIVERSITY
UNIVERSITY OF OREGON

UNIVERSITY OF SOUTHERN CALIFORNIA

STANFORD UNIVERSITY

UNIVERSITY OF HAWAII

UNIVERSITY OF TOKYO

UNIVERSITY OF UTAH

WASHINGTON STATE UNIVERSITY

UNIVERSITY OF WASHINGTON 


\section{Pacific Journal of Mathematics}

\section{Vol. 113, No. $1 \quad$ March, 1984}

Flavio E. A. da Silveira, Rational homotopy theory of fibrations $\ldots \ldots \ldots \ldots 1$

Donald M. Davis, Desuspensions of stunted projective spaces ............ 35

Lou van den Dries, Exponential rings, exponential polynomials and

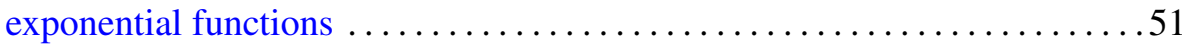

Fred Galvin and Samuel David Shore, Completeness in semimetric

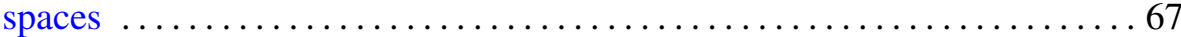

Fereidoun Ghahramani, Compact elements of weighted group algebras . . . 777

Munehiko Itōo, The closed image of a hereditary $M_{1}$-space is $M_{1} \ldots \ldots \ldots 85$

Elvira Laura Livorni, Classification of algebraic surfaces with sectional genus less than or equal to six. I. Rational surfaces .............. 93

H. Alan MacLean, Riesz sets and a theorem of Bochner ............ 115

E. Neher, Jordan triple systems with completely reducible derivation or

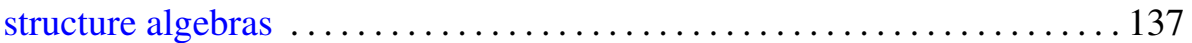

Joe Repka, Shalika's germs for $p$-adic GL( $n)$. I. The leading term $\ldots \ldots \ldots 165$

Joe Repka, Shalika's germs for $p$-adic GL( $n)$. II. The subregular term $\ldots \ldots 173$

Rae Michael Andrew Shortt, Borel density, the marginal problem and isomorphism types of analytic sets $\ldots \ldots \ldots \ldots \ldots \ldots \ldots \ldots \ldots \ldots \ldots \ldots \ldots$

Baruch Solel, The multiplicity functions of invariant subspaces for nonselfadjoint crossed products $\ldots \ldots \ldots \ldots \ldots \ldots \ldots \ldots \ldots \ldots \ldots \ldots . \ldots \ldots$

Su-win Yang, Self-intersection number of immersions and enumeration of nonstable vector bundles

W. M. Zajączkowski, Local solvability of nonstationary leakage problem for ideal incompressible fluid. II 